\title{
Use of proton pump inhibitors may cause squamous epithelial masking of intramucosal carcinoma in Barrett's esophagus
}

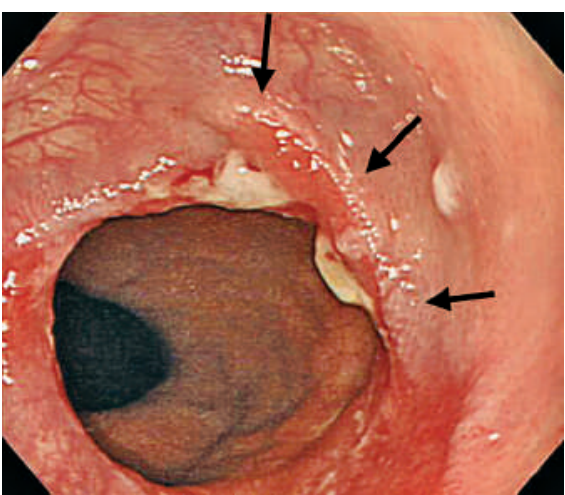

Figure 1 Endoscopy showed an erythematous irregular mucosa in the right side on the squamo-columnar junction (arrows).

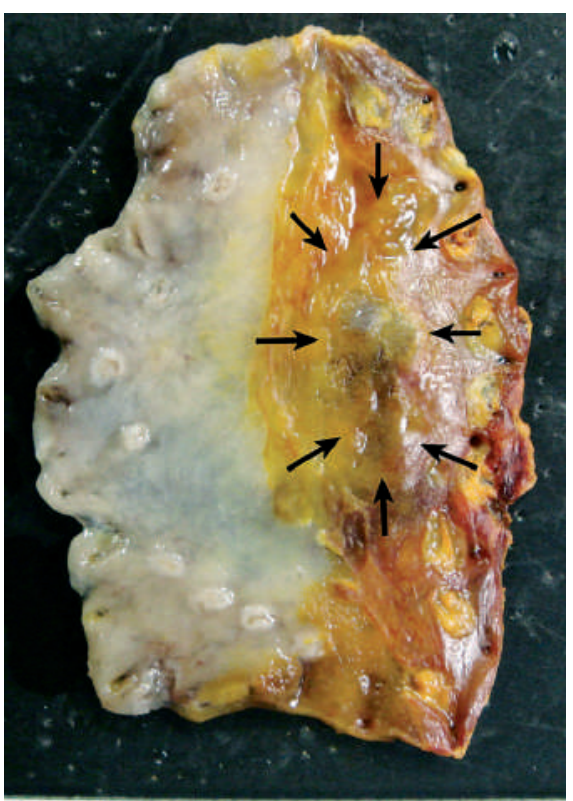

||||||||||||||||||||||||||

Figure 4 The resected specimen showed that the lesion was in the iodine-staining mucosa (arrows).

We present here a rare case of intramucosal carcinoma arising in Barrett's esophagus, but masked with squamous epithelium because of administration of a proton pump inhibitor.

A 70-year-old man underwent gastrointestinal endoscopy as a follow-up examination of gastroesophageal reflux disease. Endoscopy showed an erythematous irregular mucosa with white plaques, cov-

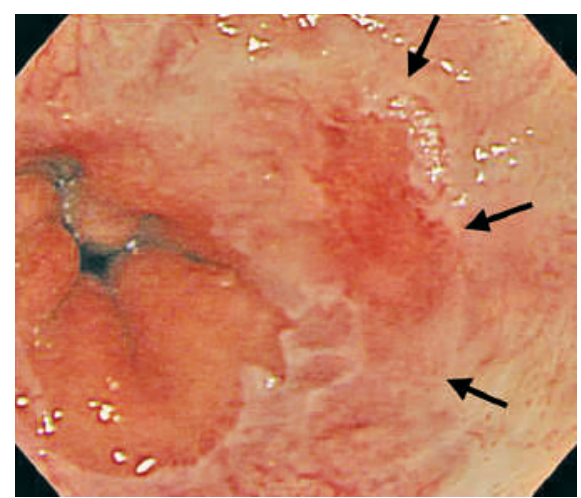

Figure 2 Five weeks after starting administration of a proton pump inhibitor, endoscopy showed that the lesion was mildly erythematous, and the inflammation had improved. Most of the lesion was covered with squamous epithelium, and the demarcation was unclear (arrows).
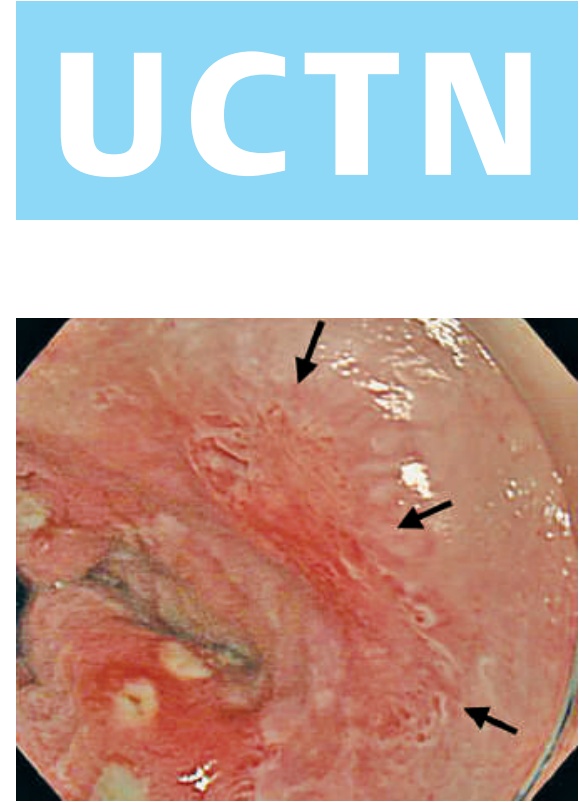

Figure 3 The endoscopic image using acetic acid instillation revealed more clearly that the tumor was covered with squamous epithelium (arrows).

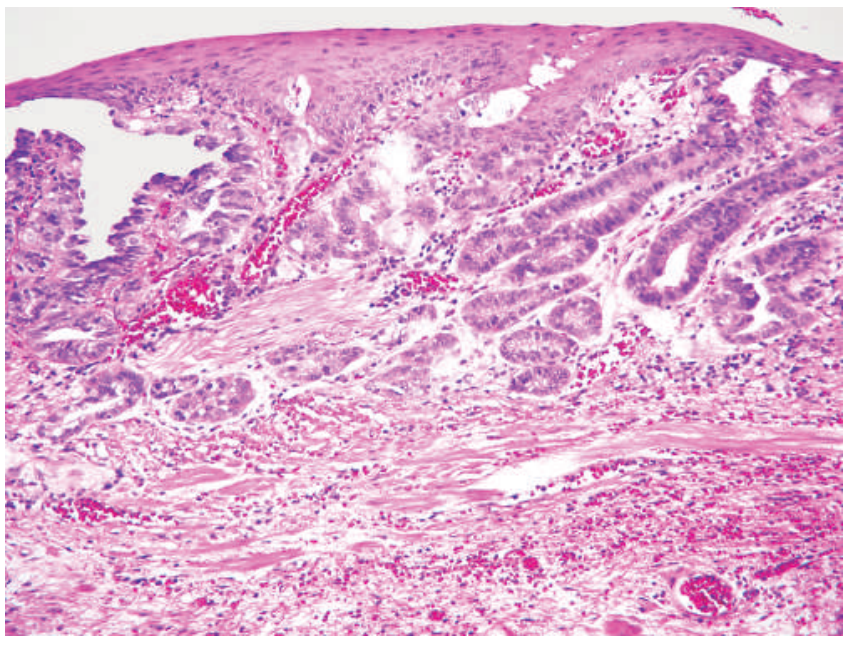

ering a quarter of the circumference in the right side on the squamo-columnar junction (Figure 1). Histopathologically, the biopsy specimen of the lesion revealed an adenocarcinoma. Esophagectomy was proposed, but it was refused by the patient. Because the inflammation of the lesion might be too severe to perform endoscopic mucosectomy, the patient was started on $20 \mathrm{mg}$ rabeprazole daily.

Five weeks after starting the proton pump inhibitor, endoscopy showed marked improvement of the inflammation (Figure 2). Most of the lesion was covered with squamous epithelium, and the demarcation was unclear. The endoscopic
Figure 5 High-magnification microscopic images show well-differentiated adenocarcinoma beneath the squamous epithelium $(\mathrm{H} \& \mathrm{E}, \times 100)$.

image using acetic acid instillation revealed more clearly that the tumor was covered with squamous epithelium (Figure 3). Endoscopic ultrasonograpy showed that the lesion was confined to the mucosal layer. We performed an endoscopic mucosectomy, and the lesion was completely resected en bloc without complication.

The resected specimen showed that the lesion was in the iodine-staining mucosa (Figure 4). Histopathologically, the lesion

DOI: 10.1055/s-2006-945174 
was diagnosed as a well-differentiated adenocarcinoma limited to the mucosa, and most of the tumor was covered with squamous epithelium (Figure $\mathbf{5}$ ).

Intestinal metaplasia, dysplasia, or carcinoma in Barrett's esophagus can be replaced by squamous epithelium after endoscopic treatment associated with acid suppression therapy [1-5]. However, it has not been reported previously that acid suppression alone could lead to squamous re-epithelialization over a carcinoma in Barrett's esophagus. Thus the use of proton pump inhibitors should be monitored carefully because they may cause masking of a small carcinoma in Barrett's esophagus.

Endoscopy_UCTN_Code_CCL_1AB_2AC_3AB

Endoscopy_UCTN_Code_CCL_1AB_2AC_3AC

K. Tanaka ${ }^{1}$, H. Toyoda ${ }^{1}$, S. Kadowaki ${ }^{2}$, Y. Hamada ${ }^{2}$, R. Kosaka ${ }^{2}$, M. Yamanaka ${ }^{3}$, I. Imoto ${ }^{1}$

${ }^{1}$ Department of Endoscopic Medicine, Mie University School of Medicine, Tsu, Japan

2 Department of Gastroenterology, Mie University School of Medicine, Tsu, Japan

${ }^{3}$ Department of Pathology, Mie University School of Medicine, Tsu, Japan.
References

${ }^{1}$ Barham CP, Jones RL, Biddlestone LR et al. Photothermal laser ablation of Barrett's oesophagus: endoscopic and histological evidence of squamous re-epithelialisation. Gut 1997; 41: $281-284$

2 Biddlestone LR, Barham CP, Wilkinson SP et al. The histopathology of treated Barrett's esophagus: squamous reepithelialization after acid suppression and laser and photodynamic therapy. Am J Surg Pathol 1998; 22: 239-245

${ }^{3}$ Van Laethem JL, Cremer M, Peny MO et al. Eradication of Barrett's mucosa with argon plasma coagulation and acid suppression: immediate and mid term results. Gut 1998; 43: 747 - 751

${ }^{4}$ Basu KK, Pick B, Bale R et al. Efficacy and one year follow up of argon plasma coagulation therapy for ablation of Barrett's oesophagus: factors determining persistence and recurrence of Barrett's epithelium. Gut 2002; 51: $776-780$

${ }^{5}$ Satodate $\mathrm{H}$, Inoue $\mathrm{H}$, Fukami $\mathrm{N}$ et al. Squamous reepithelialization after circumferential endoscopic mucosal resection of superficial carcinoma arising in Barrett's esophagus. Endoscopy 2004; 36: 909-912
Corresponding author

\section{K. Tanaka, MD}

Department of Endoscopic Medicine Mie University School of Medicine 2-174 Edobashi

Tsu, Mie Prefecture 514-8507

Japan

Fax: $\quad+81-59-231-5200$

Email: kyosuket@qa2.so-net.ne.jp 